\title{
SPECTRUM SENSING TECHNIQUES IN COGNITIVE RADIO NETWORKS: A SURVEY
}

\author{
Mansi Subhedar ${ }^{1}$ and Gajanan Birajdar ${ }^{2}$ \\ Department of Electronics and Telecommunication Engineering, SIES Graduate School \\ of Technology, Navi Mumbai, India \\ mansi_subhedar@rediffmail.com², gajanan123@gmail.com²
}

\begin{abstract}
The growing demand of wireless applications has put a lot of constraints on the usage of available radio spectrum which is limited and precious resource. However, a fixed spectrum assignment has lead to under utilisation of spectrum as a great portion of licensed spectrum is not effectively utilised. Cognitive radio is a promising technology which provides a novel way to improve utilisation efficiency of available electromagnetic spectrum. Spectrum sensing helps to detect the spectrum holes (underutilised bands of the spectrum) providing high spectral resolution capability. In this paper, survey of spectrum sensing techniques is presented. The challenges and issues involved in implementation of spectrum sensing techniques are discussed in detail giving comparative study of various methodologies.
\end{abstract}

\section{KEYWORDS}

Cognitive Radio, Dynamic Spectrum Access, Spectrum Sensing, Signal Processing Techniques

\section{INTRODUCTION}

The available electromagnetic radio spectrum is a limited natural resource and getting crowded day by day due to increase in wireless devices and applications. It has been also found that the allocated spectrum is underutilised because of the static allocation of the spectrum. Also, the conventional approach to spectrum management is very inflexible in the sense that each wireless operator is assigned an exclusive license to operate in a certain frequency band. And, with most of the useful radio spectrum already allocated, it is difficult to find vacant bands to either deploy new services or to enhance existing ones. In order to overcome this situation, we need to come up with a means for improved utilization of the spectrum creating opportunities for dynamic spectrum access. [1] - [3].

The issue of spectrum underutilization in wireless communication can be solved in a better way using Cognitive radio $(C R)$ technology. Cognitive radios are designed in order to provide highly reliable communication for all users of the network, wherever and whenever needed and to facilitate effective utilization of the radio spectrum. Figure 1 and 2 show relatively low utilization of the licensed spectrum which is largely due to inefficient fixed frequency allocations rather than any physical shortage of spectrum. This observation has forced the regulatory bodies to search a method where secondary (unlicensed) systems are allowed to opportunistically utilize the unused primary (licensed) bands commonly referred to as white spaces. Cognitive radio can change its transmitter parameters based on interaction with environment in which it operates. Cognitive radio includes four main functional blocks: spectrum sensing, spectrum management, spectrum sharing and spectrum mobility. Spectrum sensing aims to determine spectrum availability and the 
presence of the licensed users (also known as primary users). Spectrum management is to predict how long the spectrum holes are likely to remain available for use to the unlicensed users (also called cognitive radio users or secondary users). Spectrum sharing is to distribute the spectrum holes fairly among the secondary users bearing in mind usage cost. Spectrum mobility is to maintain seamless communication requirements during the transition to better spectrum.

Among all other functions, Spectrum sensing is believed as the most crucial task to establish cognitive radio networks. The various spectrum sensing techniques includes primary transmitter detection, cooperative detection and interference detection. These are discussed and compared in detail in upcoming sections [4].

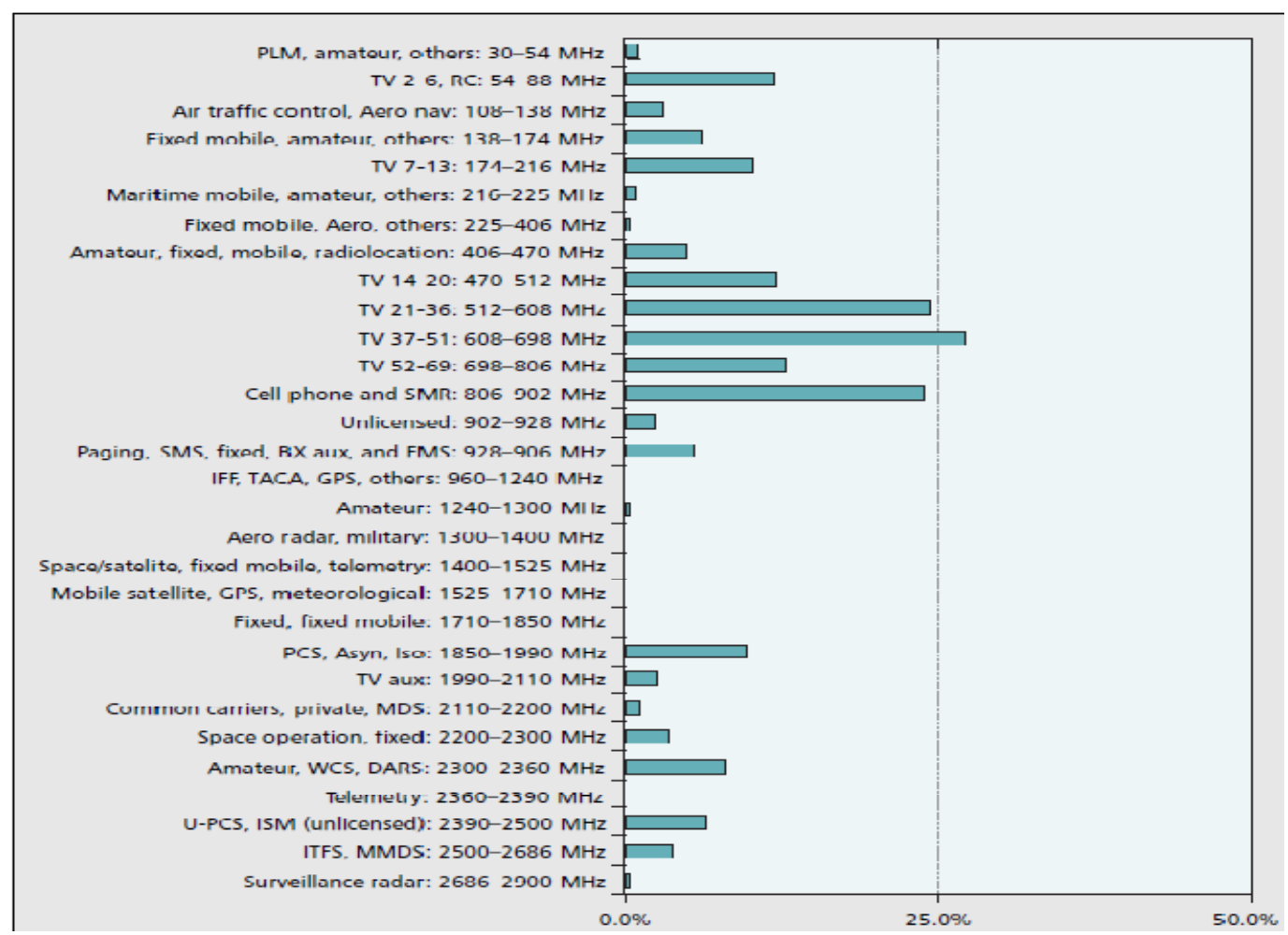

Figure1: Spectrum concentration [1]

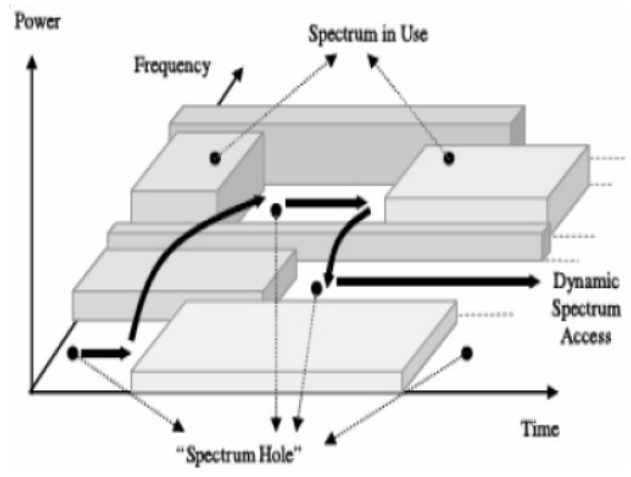

Figure 2: Illustration of spectrum white space [12] 
Organization of the paper is as follows: In section 2, various issues and challenges involved in the process of spectrum sensing are discussed. Section 3 specifies spectrum mobility issues. The need of spectrum sensing and the approaches to its use are described in section 4. Section 5 illustrates classification of various spectrum sensing techniques and in detail comparison. The paper concludes with a summary in section 6 .

\section{Issues and Challenges in Spectrum Sensing}

Several sources of uncertainty such as channel uncertainty, noise uncertainty, sensing interference limit etc. need to be addressed while solving the issue of spectrum sensing in cognitive radio networks. These issues are discussed in details as follows [5], [8].

\subsection{Channel Uncertainty}

In wireless communication networks, uncertainties in received signal strength arises due to channel fading or shadowing which may wrongly interpret that the primary system is located out of the secondary user's interference range as the primary signal may be experiencing a deep fade or being heavily shadowed by obstacles. Therefore, cognitive radios have to be more sensitive to distinguish a faded or shadowed primary signal from a white space. Any uncertainty in the received power of the primary signal translates into a higher detection sensitivity requirement. Figure 3 shows the trade off between spectrum sensing time and user throughput.
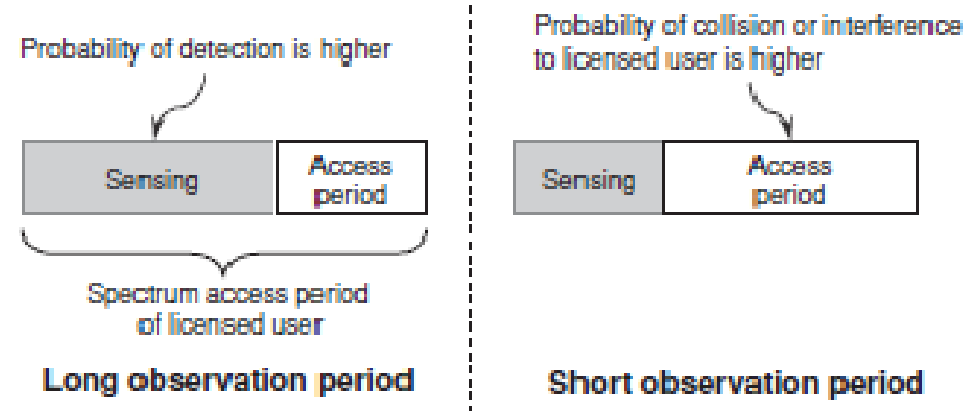

Figure 3: Trade off between spectrum sensing time and user throughput [6]

Under severe fading, a single cognitive radio relying on local sensing may be unable to achieve this increased sensitivity since the required sensing time may exceed the sensing period. This issue may be handled by having a group of cognitive radios (cooperative Sensing), which share their local measurements and collectively decide on the occupancy state of a licensed band.

\subsection{Noise Uncertainty}

The detection sensitivity can be defined as the minimum SNR at which the primary signal can be accurately (e.g. with a probability of 0.99 ) detected by the cognitive radio and is given by [5],

$$
\gamma_{\min }=\frac{P_{p} L(D+R)}{N}
$$

Where $\mathrm{N}$ is the noise power, $\mathrm{Pp}$ is transmitted power of the primary user, $\mathrm{D}$ is the interference range of the secondary user, and $\mathrm{R}$ is maximum distance between primary transmitter and its corresponding receiver. 
The above equation suggests that in order to calculate the required detection sensitivity, the noise power has to be known, which is not available in practice, and needs to be estimated by the receiver. However the noise power estimation is limited by calibration errors as well as changes in thermal noise caused by temperature variations. Since a cognitive radio may not satisfy the sensitivity requirement due to an underestimate of $\mathrm{N}, \gamma_{\min }$ should be calculated with the worst case noise assumption, thereby necessitating a more sensitive detector [20].

\subsection{Aggregate Interference Uncertainty}

In future, due to the widespread deployment of secondary systems, there will be increased possibility of multiple cognitive radio networks operating over the same licensed band. As a result, spectrum sensing will be affected by uncertainty in aggregate interference (e.g. due to the unknown number of secondary systems and their locations). Though, a primary system is out of interference range of a secondary system, the aggregate interference may lead to wrong detection. This uncertainty creates a need for more sensitive detector, as a secondary system may harmfully interfere with primary system located beyond its interference range, and hence it should be able to detect them.

\subsection{Sensing Interference Limit}

Primary goal of spectrum sensing is to detect the spectrum status i.e. whether it is idle or occupied, so that it can be accessed by an unlicensed user. The challenge lies in the interference measurement at the licensed receiver caused by transmissions from unlicensed users. First, an unlicensed user may not know exactly the location of the licensed receiver which is required to compute interference caused due to its transmission. Second, if a licensed receiver is a passive device, the transmitter may not be aware of the receiver. So these factors need attention while calculating the sensing interference limit.

\section{Spectrum Mobility Issues}

The spectrum mobility functions in a cognitive radio network allow an unlicensed user to change its operating spectrum dynamically based on the spectrum conditions. This issue can be addressed in following ways. [6]

- Search for the best frequency band: A cognitive radio must keep track of available frequency bands so that if necessary (e.g. a licensed user is detected), it can switch immediately to other frequency band. During transmission by an unlicensed user, the condition of the frequency band has to be observed. In a similar way to spectrum sensing, this would of course incur some overhead. The observation can be performed in a proactive manner or in an on demand basis. In the proactive approach, the condition of the available channels is periodically observed and the knowledge about these channels is continuously updated. In an on demand approach, channel observation can be performed only when an unlicensed user needs to switch the channel.

- Protocol stack adaptation: Since the latency due to spectrum handoff could be high, the modification and adaptation of other components in the protocol stack is required. For example, when an unlicensed user switches channel, the TCP timer at the transport layer can be frozen to avoid any miss interpretation of the delay incurred for the acknowledgement message. A cross layer optimized framework for protocol adaptation has to be developed to cope up with spectrum mobility.

- Self coexistence and synchronization: When an unlicensed (or secondary) user performs spectrum handoff, two issues have to be taken into account [22]. First, the target channel must not currently be used by any other secondary user (i.e. the self coexistence requirement), and the receiver of the corresponding secondary link must be notified of the spectrum handoff (i.e. the synchronization requirement). For the self coexistence issue, a spectrum broker can be used to 
manage spectrum allocation. For synchronization, the MAC protocol must be designed with provision for spectrum handoff information exchange.

\section{Spectrum Sensing}

A major challenge in cognitive radio is that the secondary users need to detect the presence of primary users in a licensed spectrum and quit the frequency band as quickly as possible if the corresponding primary radio emerges in order to avoid interference to primary users. This technique is called spectrum sensing. Spectrum sensing and estimation is the first step to implement Cognitive Radio system [5].

We can categorize spectrum sensing techniques into direct method, which is considered as frequency domain approach, where the estimation is carried out directly from signal and indirect method, which is known as time domain approach, where the estimation is performed using autocorrelation of the signal. Another way of categorizing the spectrum sensing and estimation methods is by making group into model based parametric method and periodogram based nonparametric method.

Another way of classification depends on the need of spectrum sensing as stated below [13]:

\subsection{Spectrum Sensing for Spectrum Opportunities}

a. Primary transmitter detection: In this case, the detection of primary users is performed based on the received signal at CR users. This approach includes matched filter (MF) based detection, energy based detection, covariance based detection, waveform based detection, cyclostationary based detection, radio identification based detection and random Hough Transform based detection.

b. Cooperative and collaborative detection: In this approach, the primary signals for spectrum opportunities are detected reliably by interacting or cooperating with other users, and the method can be implemented as either centralized access to spectrum coordinated by a spectrum server or distributed approach implied by the spectrum load smoothing algorithm or external detection.

\subsection{Spectrum Sensing for Interference Detection}

a. Interference temperature detection: In this approach, CR system works as in the ultra wide band (UWB) technology where the secondary users coexist with primary users and are allowed to transmit with low power and are restricted by the interference temperature level so as not to cause harmful interference to primary users.

b. Primary receiver detection: In this method, the interference and/or spectrum opportunities are detected based on primary receiver's local oscillator leakage power. 


\section{Classification of Spectrum Sensing Techniques}

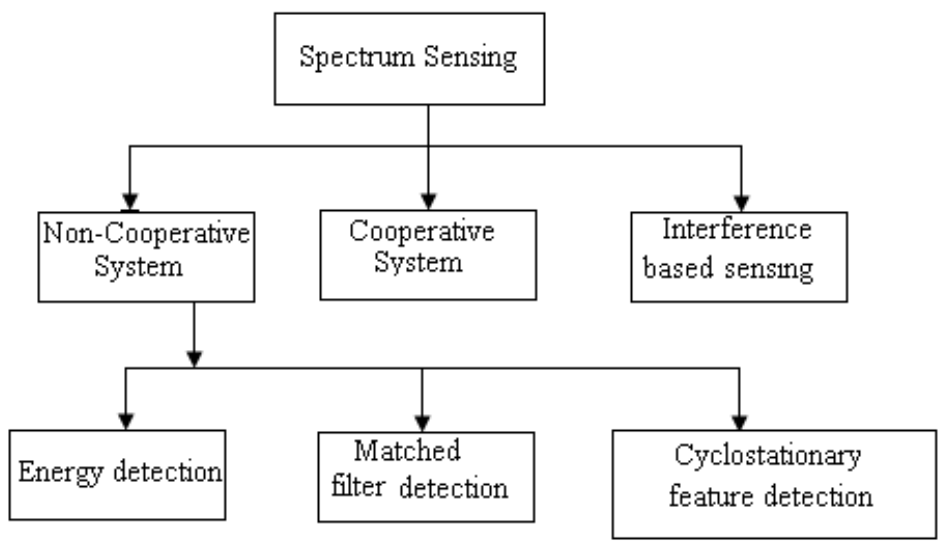

Figure 4: Classification of spectrum sensing techniques [6]

Figure 4 shows the detailed classification of spectrum Sensing techniques. They are broadly classified into three main types, transmitter detection or non cooperative sensing, cooperative sensing and interference based sensing. Transmitter detection technique is further classified into energy detection, matched filter detection and cyclostationary feature detection [14].

\subsection{Primary Transmitter Detection}

\subsubsection{Energy Detection}

It is a non coherent detection method that detects the primary signal based on the sensed energy [1]. Due to its simplicity and no requirement on a priori knowledge of primary user signal, energy detection (ED) is the most popular sensing technique in cooperative sensing [15]-[17].

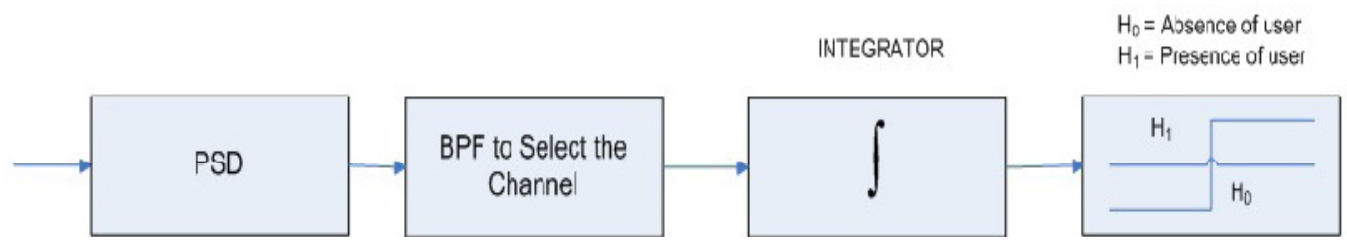

Figure 5: Energy detector block diagram [1]

The block diagram for the energy detection technique is shown in the Figure 5. In this method, signal is passed through band pass filter of the bandwidth $\mathrm{W}$ and is integrated over time interval. The output from the integrator block is then compared to a predefined threshold. This comparison is used to discover the existence of absence of the primary user. The threshold value can set to be fixed or variable based on the channel conditions.

The ED is said to be the Blind signal detector because it ignores the structure of the signal. It estimates the presence of the signal by comparing the energy received with a known threshold $v$ derived from the statistics of the noise. Analytically, signal detection can be reduced to a simple identification problem, formalized as a hypothesis test, 


$$
\begin{aligned}
& y(k)=n(k) \ldots \ldots \ldots \ldots \ldots \ldots \ldots H_{0} \\
& y(k)=h * s(k)+n(k) \ldots \ldots \ldots H_{1}
\end{aligned}
$$

Where $\mathrm{y}(\mathrm{k})$ is the sample to be analyzed at each instant $\mathrm{k}$ and $\mathrm{n}(\mathrm{k})$ is the noise of variance $\sigma^{2}$. Let $\mathrm{y}(\mathrm{k})$ be a sequence of received samples $\mathrm{k} \in\{1,2 \ldots \mathrm{N}\}$ at the signal detector, then a decision rule can be stated as,

$$
\begin{aligned}
& H_{0} \ldots \text {.if } \varepsilon<v \\
& H_{1} \ldots i f \varepsilon>v
\end{aligned}
$$

where $\varepsilon=E|y(k)|^{2}$ the estimated energy of the received signal and $v$ is chosen to be the noise variance $\sigma^{2}$.

However ED is always accompanied by a number of disadvantages i) sensing time taken to achieve a given probability of detection may be high. ii) detection performance is subject to the uncertainty of noise power. iii) ED cannot be used to distinguish primary signals from the CR user signals. As a result $\mathrm{CR}$ users need to be tightly synchronized and refrained from the transmissions during an interval called Quiet Period in cooperative sensing. iv) ED cannot be used to detect spread spectrum signals [9].

\subsubsection{Matched Filter}

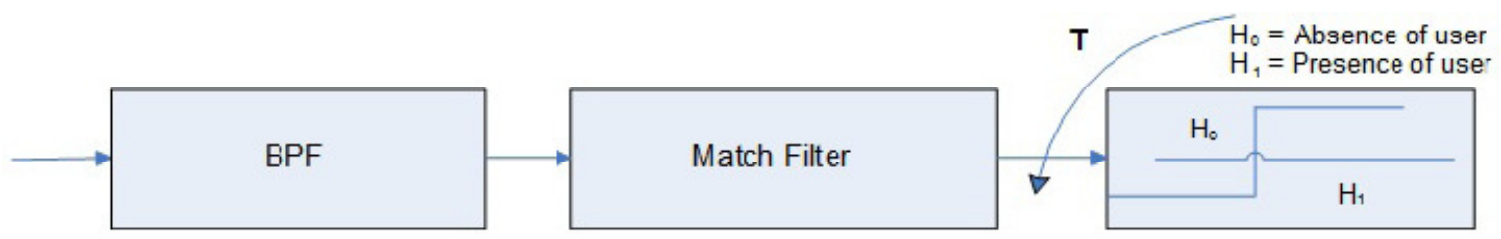

Figure 6: Block diagram of matched filter [1]

A matched filter (MF) is a linear filter designed to maximize the output signal to noise ratio for a given input signal. When secondary user has a priori knowledge of primary user signal, matched filter detection is applied. Matched filter operation is equivalent to correlation in which the unknown signal is convolved with the filter whose impulse response is the mirror and time shifted version of a reference signal. The operation of matched filter detection is expressed as:

$$
Y[n]=\sum_{K=-\infty}^{\infty} h[n-k] x[k]
$$

Where ' $x$ ' is the unknown signal (vector) and is convolved with the ' $h$ ', the impulse response of matched filter that is matched to the reference signal for maximizing the SNR. Detection by using matched filter is useful only in cases where the information from the primary users is known to the cognitive users.

Advantages: Matched filter detection needs less detection time because it requires only $\mathrm{O}$ (1/SNR) samples to meet a given probability of detection constraint. When the information of the primary user signal is known to the cognitive radio user, matched filter detection is optimal detection in stationary gaussian noise [9]. 
Disadvantages: Matched filter detection requires a prior knowledge of every primary signal. If the information is not accurate, MF performs poorly. Also the most significant disadvantage of MF is that a CR would need a dedicated receiver for every type of primary user.

\subsubsection{Cyclostationary Feature Detection}

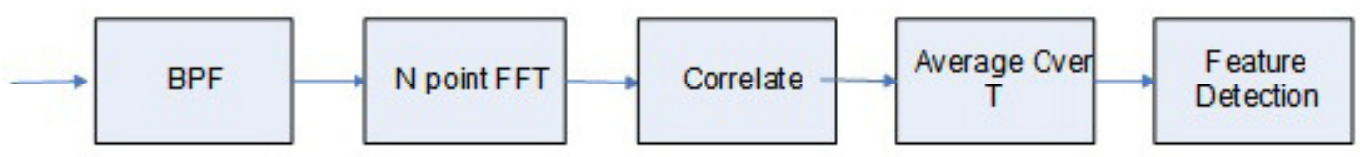

Figure 7: Cyclostationary feature detector block diagram [1]

It exploits the periodicity in the received primary signal to identify the presence of primary users (PU). The periodicity is commonly embedded in sinusoidal carriers, pulse trains, spreading code, hopping sequences or cyclic prefixes of the primary signals. Due to the periodicity, these cyclostationary signals exhibit the features of periodic statistics and spectral correlation, which is not found in stationary noise and interference [19].

Thus, cyclostationary feature detection is robust to noise uncertainties and performs better than energy detection in low SNR regions. Although it requires a priori knowledge of the signal characteristics, cyclostationary feature detection is capable of distinguishing the CR transmissions from various types of PU signals. This eliminates the synchronization requirement of energy detection in cooperative sensing. Moreover, CR users may not be required to keep silent during cooperative sensing and thus improving the overall CR throughput. This method has its own shortcomings owing to its high computational complexity and long sensing time. Due to these issues, this detection method is less common than energy detection in cooperative sensing [21].

The comparison of different transmitter detection techniques for spectrum sensing and the spectrum opportunities is shown in figure 8 . As it is evident from the figure, that matched filter based detection is complex to implement in CRs, but has highest accuracy. Similarly, the energy based detection is least complex to implement in CR system and least accurate compared to other approaches. And other approaches are in the middle of these two. 


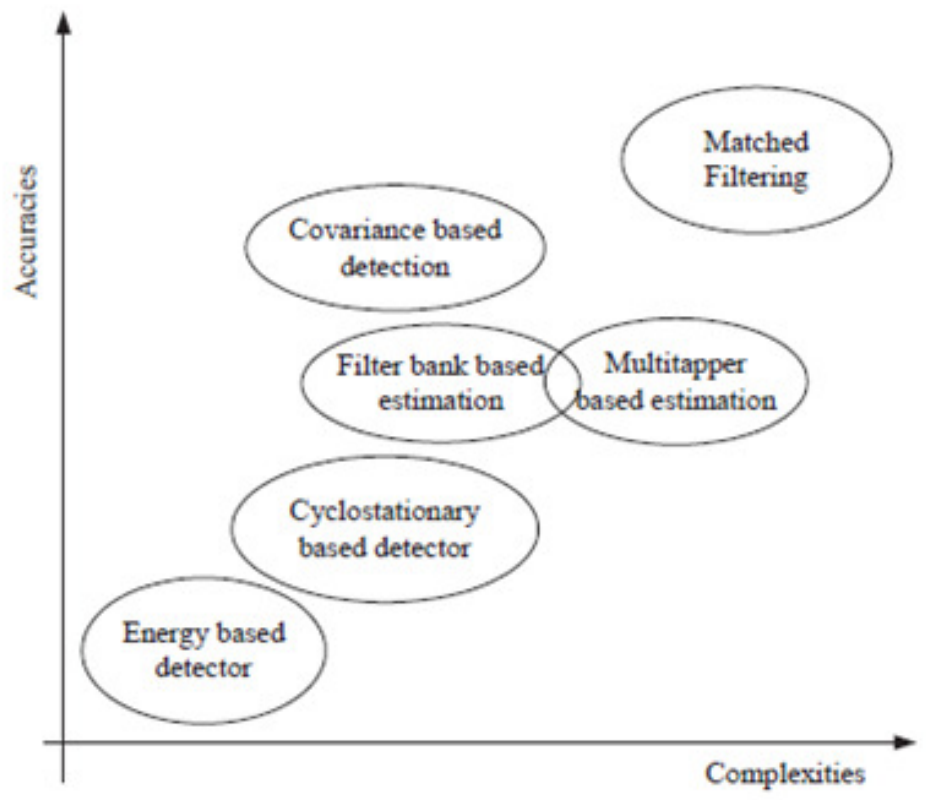

Figure 8: Sensing accuracy and complexity of various sensing methods

\subsection{Cooperative Techniques}

High sensitivity requirements on the cognitive user can be alleviated if multiple CR users cooperate in sensing the channel. Various topologies are currently used and are broadly classifiable into three regimes according to their level of cooperation [9], [25]-[26], [28].

a

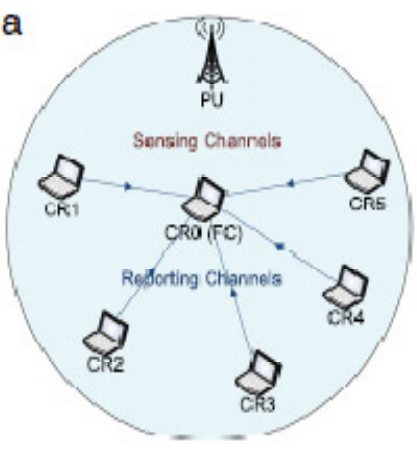

b

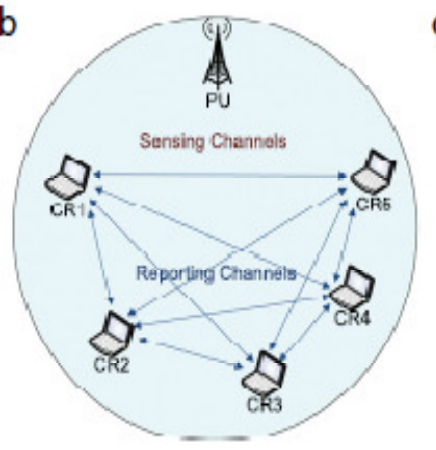

c

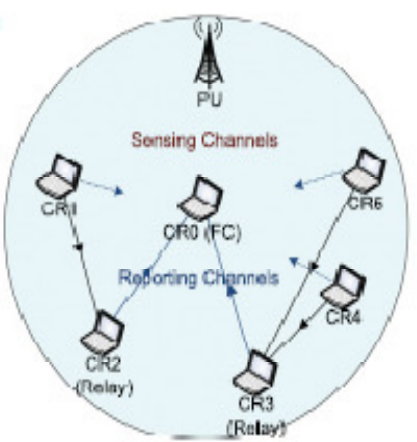

Figure 9: Cooperative sensing techniques: a-Centralised Coordinated, b- Decentralised Coordinated, and c-Decentralised Uncoordinated [9], [29].

5.2.1 Decentralized Uncoordinated Techniques: The cognitive users in the network don't have any kind of cooperation which means that each CR user will independently detect the channel, and if a CR user detects the primary user it would vacate the channel without informing the other users. Uncoordinated techniques are fallible in comparison with coordinated techniques. Therefore, CR users that experience bad channel realizations detect the channel incorrectly thereby causing interference at the primary receiver. 
5.2.2 Centralized Coordinated Techniques: In such networks, an infrastructure deployment is assumed for the CR users. One CR that detects the presence of a primary transmitter or receiver, informs a CR controller which can be a wired immobile device or another CR user. The CR controller notifies all the $\mathrm{CR}$ users in its range by means of a broadcast control message. Centralized schemes can be further classified according to their level of cooperation as: Partially cooperative where network nodes cooperate only in sensing the channel. CR users independently detect the channel and inform the CR controller which then notifies all the CR users; and totally cooperative Schemes where nodes cooperate in relaying each other's information in addition to cooperatively sensing the channel [27].

5.2.3 Decentralized Coordinated Techniques: This type of coordination implies building up a network of cognitive radios without having the need of a controller. Various algorithms have been proposed for the decentralized techniques among which are the gossiping algorithms or clustering schemes, where cognitive users gather to clusters, auto coordinating themselves [23]. The cooperative spectrum sensing raises the need for a control channel, which can be implemented as a dedicated frequency channel or as an underlay UWB channel.

5.2.4 Benefits of Cooperation: Cognitive users selflessly cooperating to sense the channel have lot of benefits among which the plummeting sensitivity requirements :channel impairments like multipath fading, shadowing and building penetration losses, impose high sensitivity requirements inherently limited by cost and power requirements. Employing cooperation between nodes can drastically reduce the sensitivity requirements up to $-25 \mathrm{dBm}$, also reduction in sensitivity threshold can be obtained by using this scheme; agility improvement: all topologies of cooperative networks reduce detection time compared to uncoordinated networks[9].

5.2.5 Disadvantages of Cooperation: The $C R$ users need to perform sensing at periodic intervals as sensed information become obsolete fast due to factors like mobility, channel impairments etc.

This considerably increases the data overhead; large sensory data: since the cognitive radio can potentially use any spectrum hole, it will have to scan a wide range of spectrum, resulting in large amounts of data, being inefficient in terms of data throughput, delay sensitivity requirements and energy consumption. Even though cooperatively sensing data poses lot of challenges, it could be carried out without incurring much overhead because only approximate sensing information is required, eliminating the need for complex signal processing schemes at the receiver and reducing the data load. Also, even though a wide channel has to be scanned, only a portion of it changes at a time requiring updating only the changed information and not all the details of the entire scanned spectrum [9-12].

\subsection{Interference Based Detection}

In this section, we present interference based detection so that the CR users would operate in spectrum underlay (UWB like) approach.

\subsubsection{Primary Receiver Detection}

In general, primary receiver emits the local oscillator (LO) leakage power from its RF front end while receiving the data from primary transmitter. It has been suggested as a method to detect primary user by mounting a low cost sensor node close to a primary user's receiver in order to detect the local oscillator (LO) leakage power emitted by the RF front end of the primary user's receiver which are within the communication range of CR system users. The local sensor then 
reports the sensed information to the CR users so that they can identify the spectrum occupancy status. We note that this method can also be used to identify the spectrum opportunities to operate $\mathrm{CR}$ users in spectrum overlay.

\subsubsection{Interference Temperature Management}

Unlike the primary receiver detection, the basic idea behind the interference temperature management is to set up an upper interference limit for given frequency band in specific geographic location such that the CR users are not allowed to cause harmful interference while using the specific band in specific area. Typically, CR user transmitters control their interference by regulating their transmission power (their out of band emissions) based on their locations with respect to primary users. This method basically concentrates on measuring interference at the receiver [24].

The operating principle of this method is like an UWB technology where the CR users are allowed to coexist and transmit simultaneously with primary users using low transmit power that is restricted by the interference temperature level so as not to cause harmful interference to primary users.

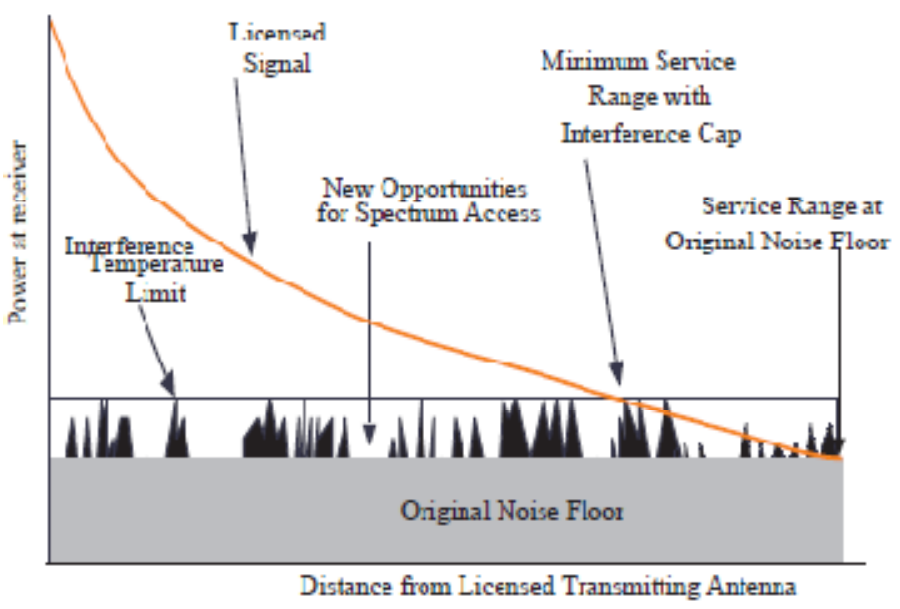

Figure 10: Interference temperature model [6]

Here CR users do not perform spectrum sensing for spectrum opportunities and can transmit right way with specified preset power mask. However, the CR users can not transmit their data with higher power even if the licensed system is completely idle since they are not allowed to transmit with higher than the preset power to limit the interference at primary users. It is noted that the CR users in this method are required to know the location and corresponding upper level of allowed transmit power levels. Otherwise they will interfere with the primary user transmissions.

\subsection{Other Signal Processing Approaches}

5.4.1 Multi -Taper Spectrum Sensing and Estimation: Multi Taper spectrum estimation (MTSE) has proposed by Thomson (1982) before the CR concept was introduced. In this method, 
the last $\mathrm{N}$ received samples are collected in a vector form and are represented as a set of slepian base vectors. The main idea of this method is that the Fourier transforms of Slepian vectors have the maximal energy concentration in the bandwidth $f_{c}-W$ to $f_{c}+W$ under finite sample size constraints. By exploiting this feature, CR user can easily identify the spectrum opportunities in given band. As MTSE uses multiple prototype filters and is better for small sample spaces since the computational complexity increases with large number of samples.

5.4.2 Filter Bank Based Spectrum Sensing: Filter bank based spectrum estimation (FBSE) is regarded as the simplified version of MTSE which uses only one prototype filter for each band and has been proposed for multi-carrier modulation based CR systems by using a pair of matched root Nyquist filter. FBSE uses the same concept of maximal energy concentration in the bandwidth $f_{c}-W$ to $f_{c}+W$. By exploiting this information, CR user identifies the spectrum occupancy and hence the spectrum opportunities. MTSE is better for small samples whereas FBSE is better for large number of samples [7].

5.4.3 Wavelet based detection: It is widely used technique in image processing for edge detection applications. Tian and Giannakis (2006) have proposed this approach in spectrum sensing where wavelets are used for detecting edges in the power spectral density (PSD) of a wideband channel. The edges in power spectral density are the boundary between spectrum holes and occupied bands and hence it helps to find vacant bands. Based on this information CR can identify the spectrum opportunities.

5.4.4 Random Hough transform based detection: It is also widely used for pattern (such as lines, circles) detection in image processing applications. Challapali et al. (2004) have proposed to perform Random Hough transform of received signal $r(n)$ to identify the presence of radar pulses in the operating channels of IEEE 802.11 wireless systems.

5.4.5 Radio identification based detection: These techniques are used in the context of European Transparent Ubiquitous Terminal (TRUST) project (Farnham et al., 2000) which is based on several extracted features such as transmission frequency, transmission range, modulation technique etc. Once the features are extracted from the received signal, CR users exploit those features and can select suitable transmission parameters for them.

\section{CONCLuSion}

Spectrum is a very valuable resource in wireless communication systems and it has been a major research topic from last several decades. Cognitive radio is a promising technology which enables spectrum sensing for opportunistic spectrum usage by providing a means for the use of white spaces. Considering the challenges raised by cognitive radios, the use of spectrum sensing method appears as a crucial need to achieve satisfactory results in terms of efficient use of available spectrum and limited interference with the licensed primary users.

As described in this paper, the development of the cognitive radio network requires the involvement and interaction of many advanced techniques, including distributed spectrum sensing, interference management, cognitive radio reconfiguration management, and cooperative communications. Furthermore, in order to fully realize the CR system in wireless communications for efficient utilization of scarce RF spectrum, the method used in identifying the interference and/or spectrum sensing should be reliable and prompt so that the primary user will not suffer from CR system to utilize their licensed spectrum. 
We presented the different signal processing methods by grouping them into three basic groups and their details in turn. We have also presented the pros and cons of different spectrum sensing methods, and performed the comparison in terms of operation, accuracies, complexities and implementations. There exist number of issues to be addressed in terms of primary signal detection time, hardware requirements and computational complexities.

\section{REFERENCES}

[1] Shahzad A. et. al. (2010), "Comparative Analysis of Primary Transmitter Detection Based Spectrum Sensing Techniques in Cognitive Radio Systems," Australian Journal of Basic and Applied Sciences, 4(9), pp: 4522-4531, INSInet Publication.

[2] Weifang Wang (2009), “Spectrum Sensing for Cognitive Radio”, Third International Symposium on Intelligent Information Technology Application Workshops, pp: 410-412.

[3] V. Stoianovici, V. Popescu, M. Murroni (2008), "A Survey on spectrum sensing techniques in cognitive radio" Bulletin of the Transilvania University of Braşov, Vol. 15 (50).

[4] Tevfik Yucek and Huseyin Arslan (2009), "A Survey of Spectrum Sensing Algorithms for Cognitive Radio Applications", IEEE Communication Surveys \& Tutorials, VOL. 11, NO. 1, pp: 116-130.

[5] D. B. Rawat, G. Yan, C. Bajracharya (2010), "Signal Processing Techniques for Spectrum Sensing in Cognitive Radio Networks', International Journal of Ultra Wideband Communications and Systems, Vol. x, No. x/x, pp:1-10.

[6] Ekram Hossain, Dusit Niyato, Zhu Han (2009), "Dynamic Spectrum Access and Management in Cognitive Radio Networks", Cambridge University Press.

[7] D.D.Ariananda, M.K.Lakshmanan, H.Nikookar (2009), “A Survey on Spectrum Sensing techniques for Cognitive Radio”, Wireless VITAE'09, Aalborg, Denmark, pp: 74-79.

[8] Amir Ghasemi, Elvino S. Sousa (2008), "Spectrum Sensing in Cognitive Radio Networks: Requirements, Challenges and Design Trade-offs Cognitive radio communication and networks", IEEE Communication Magazine, pp: 32-39.

[9] Ian F. Akyildiz, Brandon F. Lo, Ravikumar (2011), "Cooperative spectrum sensing in cognitive radio networks: A survey, Physical Communication”, pp: 40-62.

[10] Yonghong Zeng, Ying Chang Liang, Anh Tuan Hoang, and Rui Zhang (2010), “A Review on Spectrum Sensing for Cognitive Radio: Challenges and Solutions", EURASIP Journal on Advances in Signal Processing Volume 2010, Article ID 381465, pp: 1-15.

[11] Beibei Wang and K. J. Ray Liu (2011), "Advances in Cognitive Radio Networks: A Survey" IEEE Journal of Selected topics in Signal processing, VOL.5, NO.1, pp: 5-23.

[12] Simon Haykin, David J. Thomson, and Jeffrey H. Reed (2009), "Spectrum Sensing for Cognitive Radio', IEEE Proceeding, Vol. 97, No.5, pp: 849-877.

[13] Bruce A. Fette, (2006), Cognitive Radio Technology, Newnes Publisher.

[14] Takeshi Ikuma and Mort Naraghi-Pour (2008), “A Comparison of Three Classes of Spectrum Sensing Techniques”, IEEE GLOBECOM proceedings. 
International Journal of Next-Generation Networks (IJNGN) Vol.3, No.2, June 2011

[15] Ekram Hossain, Vijay Bhargava (2007), "Cognitive Wireless Communication Networks", Springer.

[16] Linda Doyle (2009), Essentials of Cognitive Radio, Cambridge University Press.

[17] D. Cabric, A. Tkachenko, and R. Brodersen, (2006) "Spectrum sensing measurements of pilot, energy and collaborative detection," in Proc. IEEE Military Commun. Conf., Washington, D.C., USA, pp: 1-7.

[18] S. t. B. S. M. Mishra, R. Mahadevappa, and R. W. Brodersen (2007), "Cognitive technology for Ultra wideband / WiMAX coexistence," in Proc. IEEE Int. Symposium on New Frontiers in Dynamic Spectrum Access Networks, Dublin, Ireland, Apr. pp: 179-186.

[19] A. Tkachenko, D. Cabric, and R. W. Brodersen, (2007), "Cyclostationary feature detector experiments using reconfigurable BEE2," in Proc. IEEE Int. Symposium on New Frontiers in Dynamic Spectrum Access Networks, Dublin, Ireland, Apr, pp: 216-219.

[20] R. Tandra and A. Sahai, (2005), "Fundamental limits on detection in low SNR under noise uncertainty," in Proc. IEEE Int. Conf. Wireless Networks, Commun And Mobile Computing, vol. 1, Maui, HI, June , pp: 464-469.

[21] R. Tandra and A. Sahai (2007), "SNR walls for feature detectors", in Proc. IEEE Int. Symposium on New Frontiers in Dynamic Spectrum Access Networks, Dublin, Ireland, Apr, pp: 559-570.

[22] Brodersen, R., Wolisz, A., Cabric, D., Mishra, S., Willkomm, D. (2004), "CORVUS: A Cognitive radio Approach for Usage of Virtual Unlicensed Spectrum", Berkeley Wireless Research Center, white paper.

[23] Nabeel A., Hadaller, D., Keshav S. (2006), "GUESS: Gossiping Updates for Efficient Spectrum Sensing”, ACM MobiCom Workshop, pp: 12-17.

[24] Thanayankizil L., Kailas, A., (2008), "Spectrum Sensing Techniques (II): Receiver Detection and Interference Management”, http://aravind.kailas. googlepages.com

[25] F. Zeng, Z. Tian, C. Li (2010), "Distributed compressive wideband spectrum sensing in cooperative multi- hop cognitive networks”, in: Proc. Of IEEE ICC 2010, pp: 1-5.

[26] Q. Zou, S. Zheng, A. Sayed (2009), "Cooperative spectrum sensing via sequential detection for cognitive radio networks", in: Proc. Of IEEE 10th Workshop on Signal Processing Advances in Wireless Communications, 2009. SPAWC'09, pp: 121-125.

[27] M. Di Renzo, L. Imbriglio, F. Graziosi, F. Santucci, (2009), "Distributed data fusion over correlated log- normal sensing and reporting channels: application to cognitive radio networks", IEEE Transactions on Wireless Communications pp: 5813-5821

[28] M. Di Renzo, L. Imbriglio, F. Graziosi, F. Santucci (2009), "Cooperative spectrum sensing over correlated log-normal sensing and reporting channel”, in: Proc. of IEEE GLOBECOM, pp:1-8.

[29] A. Min, K. Shin, (2009), "An optimal sensing framework based on spatial RSS profile in cognitive radio networks", in: Proc. of IEEE SECON, pp: 1-9. 


\section{Authors}

Mansi Subhedar is working as Lecturer in the department of Electroni cs \& Telecommunication Engineering at SIES Graduate School of Technology, Navi Mumbai, India. She obtained B.E. (Elect. \& Telecom) from Dr. BAM University, Aurangabad, Maharashtra and M.E. (Electronics) from Mumbai University. She has been in teaching for the past five years. She is life member of ISTE. She has attended several workshops and conferences. She has published and presented papers in various national conferences across India. Her research area includes next generation networks, sensor networks and signal processing.

Gajanan Birajdar is working as Assistant Professor in the department of Electronics \& Telecommunication Engineering at SIES Graduate School of Tech nology, Navi Mumbai, India. He obtained B.E. (Electronics) from Dr. BAM University, Aurangabad, Maharashtra and M. Tech. (Elect. \& Telecom) from Dr. BAM Technological University, Lonere, India. He has been in teaching for the past 14 years. He is life member of ISTE and IETE. He has attended several seminars and workshops. He has published papers in international journals. His area of research includes Ad hoc networks, image and speech processing.
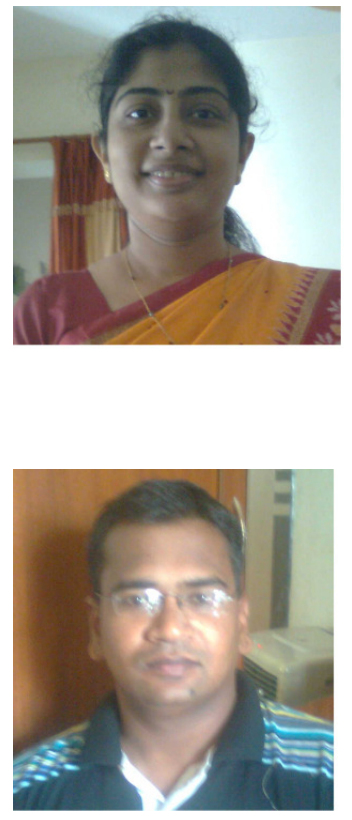